\title{
Search for squarks and gluinos in final states with jets and missing transverse momentum with the ATLAS detector
}

Christina Agapopoulou*, on behalf of the ATLAS collaboration

Laboratoire de l'Accélérateur Linéaire, France

E-mail: christina.agapopoulou@cern.ch

A search for the supersymmetric partners of quarks and gluons (squarks and gluinos) in final states containing hadronic jets and missing transverse momentum, but no electrons or muons, is presented. Starting from the definition of the analysis methodology, results obtained using data recorded by the ATLAS experiment at the Large Hadron Collider (LHC) in 2015 and 2016 (corresponding to $36.1 \mathrm{fb}^{-1}$ ) are shown.

European Physical Society Conference on High Energy Physics - EPS-HEP2019 -

10-17 July, 2019

Ghent, Belgium

${ }^{*}$ Speaker. 


\section{Introduction}

Supersymmetry (SUSY) is an extension of the Standard Model (SM) which predicts that for each fundamental particle of the Standard Model there is a new partner particle with identical quantum numbers apart from the spin. The partners of the quarks and leptons are the scalar squarks ( $\tilde{\mathrm{q}})$ and sleptons ( $(\tilde{l})$, while the gauge and Higgs bosons of the SM are partnered with new fermionic particles, the gluino $(\tilde{\mathrm{g}})$, charginos $\left(\tilde{\chi}_{i}^{ \pm}\right.$with $\left.i=1,2\right)$ and neutralinos $\left(\tilde{\chi}_{i}^{0}\right.$ with $\left.i=1,2,3,4\right)$. In the case of R-parity conservation, SUSY particles are produced and decay in pairs, with the decay chain ending at the lightest supersymmetric particle (LSP), which is stable and escapes detection, as shown in Figure 1. SUSY is a theoretically favoured extension of the SM, since it provides a possible solution to the hierarchy problem, and, in case of R-parity conservation, a dark matter candidate. The large predicted cross sections of the strong production of supersymmetric particles in high energy proton-proton collisions make gluinos and squarks a primary target for SUSY searches at the $13 \mathrm{TeV}$ proton-proton (p-p) collisions program of the ATLAS detector [1] at the Large Hadron Collider (LHC). In the following, a search for gluinos and squarks in final states with hadronic jets and missing transverse momentum, but no electrons or muons is presented. This search has been published in [2].

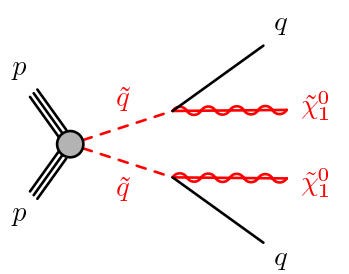

(a)

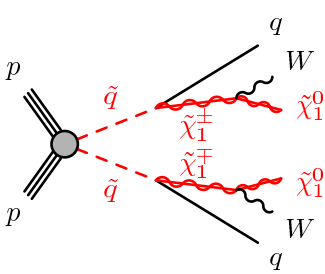

(b)

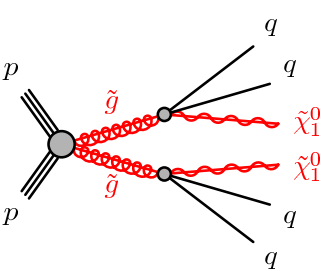

(c)

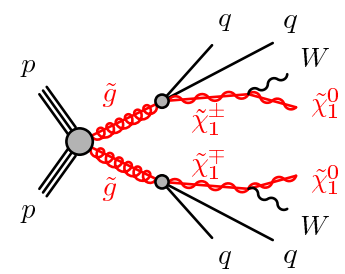

(d)

Figure 1: The decay topologies of (a,b) squark-pair production and (c,d) gluino pair production in simplified models with (a,c) showing direct decays and (b,d) showing one-step decays of squarks and gluinos.

\section{Analysis strategy}

To search for a possible signal, selection criteria are defined in order to enhance the signal yield with respect to SM backgrounds. Due to the high mass scale expected for squarks and gluinos, the main discriminating variable of this analysis is the effective mass $\left(m_{\mathrm{eff}}\right)$, defined as the scalar sum of the transverse momentum $\left(p_{\mathrm{T}}\right)$ of all jets with $p_{\mathrm{T}}>50 \mathrm{GeV}$ and of the missing transverse momentum $\left(E_{\mathrm{T}}^{\text {miss }}\right)$. Signal regions (SR) that aim to maximize the discovery sensitivity of the analysis are defined based on this variable, as well as other powerful discriminants like the jet multiplicity $\left(N_{j}\right)$, $E_{\mathrm{T}}^{\text {miss }}, E_{\mathrm{T}}^{\text {miss }} / m_{\mathrm{eff}}\left(N_{j}\right)$ and $E_{\mathrm{T}}^{\text {miss }} / \sqrt{H_{\mathrm{T}}}$, where $H_{\mathrm{T}}$ is the scalar sum of transverse momenta of all jets with $p_{\mathrm{T}}>50 \mathrm{GeV}$. Based on these variables, 24 inclusive signal regions are defined by increasing the jet multiplicity from 2 to 6, constructing the Meff-based approach. Regions with the same jet multiplicity are distinguished by varying the cut on $m_{\mathrm{eff}}$ and $E_{\mathrm{T}}^{\mathrm{miss}} / m_{\mathrm{eff}}\left(N_{j}\right)$ or $E_{\mathrm{T}}^{\mathrm{miss}} / \sqrt{H_{\mathrm{T}}}$. An alternative approach, the Recursive Jigsaw Reconstruction (RJR), is also included in the analysis. The RJR technique uses the underlying kinematic features of the event to define powerful variables 
on an event-by-event basis. A more thorough description of the application of the RJR technique on this search, as well as studies on the complementarity of the two methods can be found in [2].

\section{Background estimation}

The sensitivity of the search is limited by SM processes that produce similar signatures to the ones of the supersymmetric particles. The largest background contributions are (in decreasing order): $Z+$ jets, $W+$ jets, $t \bar{t}$, single- $t$, diboson and multi-jet production. The first four background processes are modelled with Monte-Carlo (MC) samples, while for the multi-jet background, a data driven technique [3] is used.

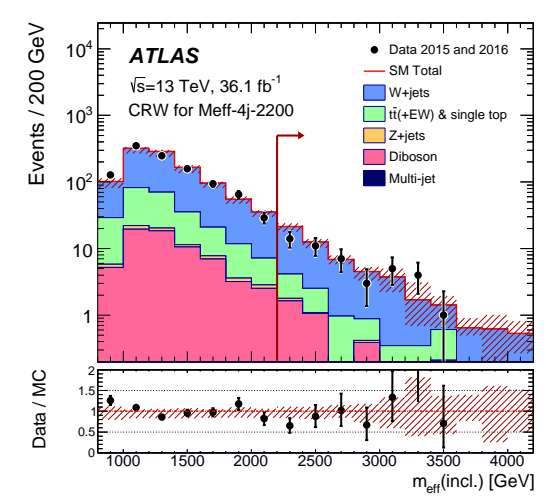

(a)

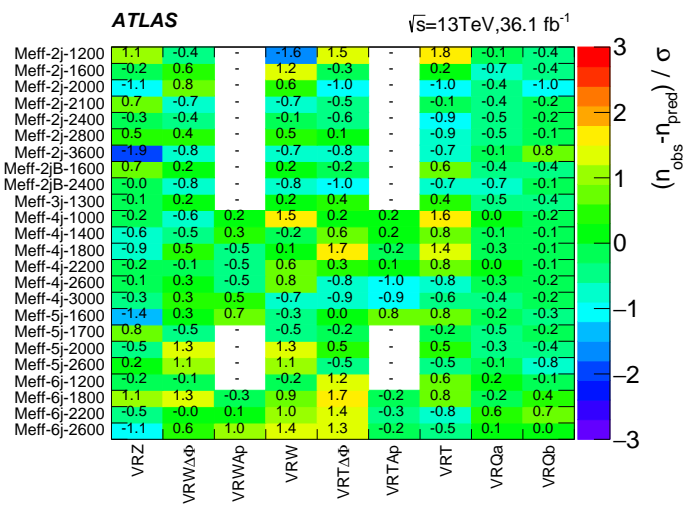

(b)

Figure 2: (a) Observed $m_{\text {eff }}$ distribution in a CRW control region [2], after applying the CR selection requirements, except from those on the plotted variable, and (b) differences between the numbers of observed events in data and the SM background predictions for each VR used in the Meff-based approach, expressed as a fraction of the total uncertainty [2].

\subsection{Control and Validation Regions}

In order to estimate the expected SM background yields, control regions (CR) are defined for each background process. The CR selections are optimized to maximize the purity and minimize the uncertainty in the targeted background, while being as close as possible to the SR phase-space, in order to reduce the extrapolation uncertainty. In the Meff-based approach, each SR has its own set of four CRs: CRY, CRW, CRT and CRQ to estimate the $Z+$ jets, $W+$ jets, $t \bar{t}+$ single- $t$ and multijet backgrounds respectively. These CRs are built using $\gamma+$ jets, $W(\rightarrow \ell v)+$ jets, $t \bar{t}$ and single- $t$ and multi-jet samples respectively. For the minor diboson background, the pure MC estimation is used.

Another set of regions, named Validation Regions (VR), is defined in order to validate the background estimation procedure for each background. These regions resemble the CRs but with kinematic cuts that drive them closer to the SR phase-space. The results of the validation procedure are shown in Figure 2b, where the difference in each VR between the numbers of observed and expected events, expressed as fractions of the one-standard deviation $(1 \sigma)$ uncertainties in the latter, are summarized. No significant biases are observed, verifying the quality of the background estimation procedure. 


\section{Fit and systematic uncertainties}

In order to perform statistical tests and extract the final results, three different types of profile likelihood fits are employed. The SM background yields in each SR are extracted from a background-only fit. The level of agreement between the SM and observation and upper limits on various SUSY models are investigated in the model independent and dependent fits, respectively. Systematic uncertainties that arise from the use of extrapolation factors relating observations in the CRs to background predictions in the SRs, and from the MC modelling of minor backgrounds are also included as nuisance parameters in the fits. These systematics uncertainties range from $6 \%$ to $67 \%$ in the different SRs and arise mainly from statistical limitations of the MC samples and theoretical uncertainties of the various models.

\section{Results, interpretations and limits}

Figure 3a shows a $m_{\text {eff }}$ distribution after applying the selection criteria of one of the signal regions, excluding the selection on the variable itself. The SM prediction is in reasonable agreement with the data, while the equivalent distribution from a supersymmetric signal sample is superimposed for comparison. In Figure 3b the observed yields per SR for the Meff-based approach along with the SM background predictions after applying the CR-derived normalization factors. In general, the data are in agreement with the expected SM background. The largest excess of the data from the SM prediction has a significance of 2.0 (2.5) $\sigma$ for the Meff-based (RJR) search.

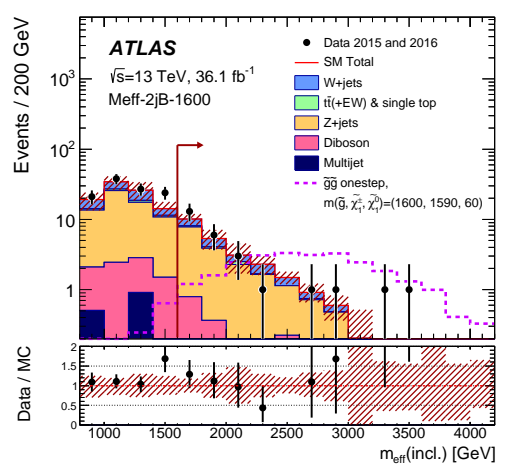

(a)

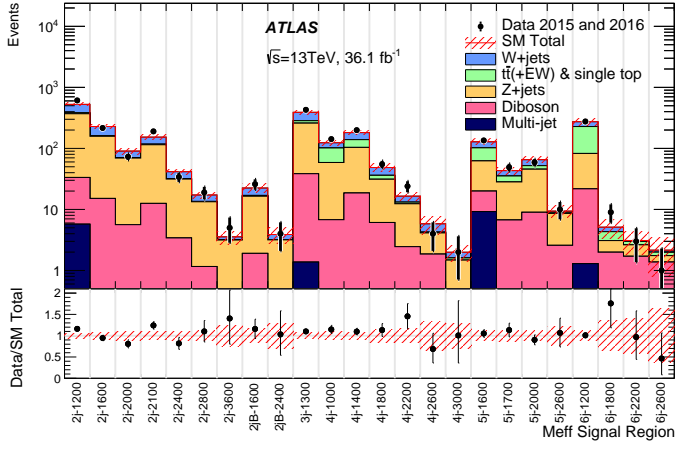

(b)

Figure 3: (a) Observed $m_{\text {eff }}$ distribution for one of the Meff-based SRs after applying all selection requirements except the ones on the plotted variable [2]. The histograms show the MC background predictions prior to the fits. (b) Comparison of the observed and expected (after the background-only fit) event yields as a function of the Meff-based SRs [2].

In the absence of statistically strong evidence for the presence of supersymmetric signals in the SRs, limits are placed on the contributions of Beyond Standard Model (BSM) physics to the SM processes using the $\mathrm{CL}_{\mathrm{s}}$ prescription. Figure 4 shows limits on the masses of SUSY particles obtained from model-dependent fits on simplified models where only direct production of first and second-generation mass-degenerate squark or gluino pairs are considered. The gluinos and squarks are treated as fully decoupled. For a massless LSP, squark (gluino) masses below 1.55 (2.03) TeV can be excluded at $95 \%$ confidence level. 


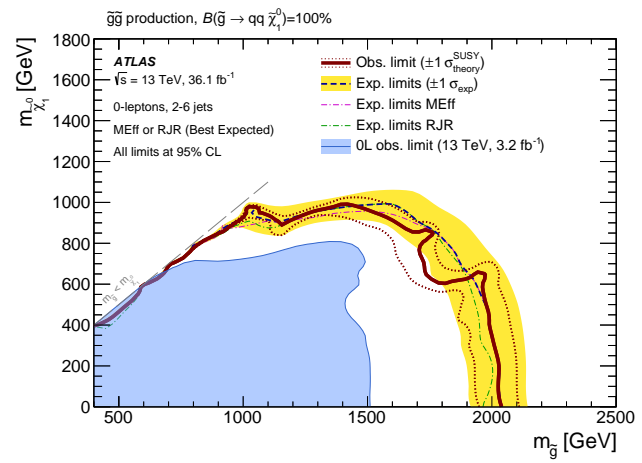

(a)

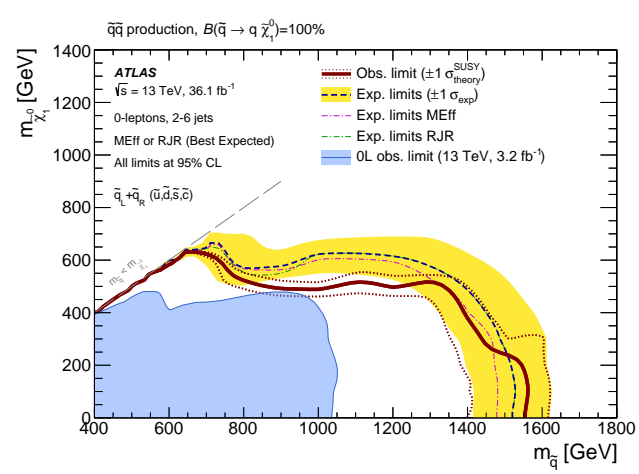

(b)

Figure 4: Exclusion limits for direct production of (a) first- and second-generation squark pairs with decoupled gluinos and (b) gluino pairs with decoupled squarks [2]. Exclusion limits are obtained by using the signal region with the best expected sensitivity at each point. Expected limits from the Meff- and RJR-based searches separately are also shown for comparison. Results are compared with the observed limits obtained by the $2015\left(3.2 \mathrm{fb}^{-1}\right)$ ATLAS searches with jets, missing transverse momentum, and no leptons.

\section{Conclusions}

The results of the search for squarks and gluinos in final states with jets, missing transverse momentum and no leptons with $36 \mathrm{fb}^{-1}$ of data from $\sqrt{\mathrm{s}}=13 \mathrm{TeV} \mathrm{p}-\mathrm{p}$ collisions recorded with the ATLAS detector at the LHC have been presented. Two complementary approaches, the Meff-based approach and the RJR technique have been deployed to maximize the discovery sensitivity of the analysis. No significant deviation from the SM background is observed. Results are interpreted in terms of simplified models with only first- and second-generation squarks and gluinos, along with a neutralino LSP, with all the other SUSY particles decoupled. For a massless LSP, squark masses below $1.55 \mathrm{TeV}$ are excluded at $95 \%$ confidence level, assuming mass-degeneracy of the two first generation squarks. For simplified models with pair produced gluinos that decay directly into quarks and the LSP, gluino masses below $2.03 \mathrm{TeV}$ are also excluded. A substantial improvement of these limits has been achieved in a recently published analysis that uses the full Run-2 dataset of the ATLAS detector [4].

\section{References}

[1] ATLAS Collaboration, The ATLAS Experiment at the CERN Large Hadron Collider, 2008 JINST 3 S08003

[2] ATLAS Collaboration, Search for squarks and gluinos in final states with jets and missing transverse momentum using $36 \mathrm{fb}^{-1}$ of $\sqrt{\mathrm{s}}=13 \mathrm{TeV}$ pp collision data with the ATLAS detector, Phys. Rev. D 97 (2018) 112001, arXiv: [1712.02332]

[3] ATLAS Collaboration, Search for squarks and gluinos with the ATLAS detector in final states with jets and missing transverse momentum using $4.7 \mathrm{fb}^{-1}$ of $\sqrt{\mathrm{s}}=7 \mathrm{TeV}$ proton-proton collision data, Phys. Rev. D 87 (2013) 012008, arXiv: [1208.0949]

[4] ATLAS Collaboration, Search for squarks and gluinos in final states with jets and missing transverse momentum using $139 \mathrm{fb}^{-1}$ of $\sqrt{\mathrm{s}}=13 \mathrm{TeV}$ pp collision data with the ATLAS detector,

ATLAS-CONF-2019-040, https://cds.cern.ch/record/2686254 